\title{
SEMIGEODESICS AND THE MINIMAL TIME FUNCTION
}

\author{
Chadi Nour ${ }^{1}$
}

\begin{abstract}
We study the Hamilton-Jacobi equation of the minimal time function in a domain which contains the target set. We generalize the results of Clarke and Nour [J. Convex Anal., 2004], where the target set is taken to be a single point. As an application, we give necessary and sufficient conditions for the existence of solutions to eikonal equations.
\end{abstract}

Mathematics Subject Classification. 49J52, 49L20, 49L25.

Received June 29, 2004. Accepted February 25, 2005.

\section{INTRODUCTION}

Let $F$ be a multivalued function mapping $\mathbb{R}^{\mathrm{n}}$ to the subsets of $\mathbb{R}^{\mathrm{n}}$. We assume throughout this paper that $F$ satisfies the standing hypotheses; that is, $F$ takes nonempty compact convex values, has closed graph, and satisfies a linear growth condition: for some positive constants $\gamma$ and $c$, and for all $x \in \mathbb{R}^{\mathrm{n}}$,

$$
v \in F(x) \Longrightarrow\|v\| \leq \gamma\|x\|+c .
$$

The multivalued function $F$ is also taken to be locally Lipschitz: every $x \in \mathbb{R}^{\mathrm{n}}$ admits a neighborhood $U=U(x)$ and a positive constant $K=K(x)$ such that

$$
x_{1}, x_{2} \in U \Longrightarrow F\left(x_{2}\right) \subseteq F\left(x_{1}\right)+K\left\|x_{1}-x_{2}\right\| \bar{B} .
$$

We associate with $F$ the following function $h$, the lower Hamiltonian:

$$
h(x, p):=\min \{\langle p, v\rangle: v \in F(x)\} .
$$

Now let $S$ be a nonempty compact subset of $\mathbb{R}^{\mathrm{n}}$, we denote by $T(\cdot, S)$ the well-known minimal time function associated to $S$ (for the dynamic $F$ ). We recall that this function is defined as follows:

$$
T(\alpha, S):=\left\{\begin{array}{l}
\inf T \geq 0, \\
\dot{x}(t) \in F(x(t)) \text { a.e. } t \in[0, T] \\
x(0)=\alpha \\
x(T) \in S .
\end{array}\right.
$$

Keywords and phrases. Minimal time function, Hamilton-Jacobi equations, viscosity solutions, minimal trajectories, eikonal equations, monotonicity of trajectories, proximal analysis, nonsmooth analysis.

${ }^{1}$ Computer Science and Mathematics Division, Lebanese American University, Byblos Campus, P.O. Box 36, Byblos, Lebanon; cnour@lau.edu.lb This paper was written while the author was an ATER at Institut Girard Desargues, Université Lyon I.

(C) EDP Sciences, SMAI 2006 
If no trajectory between $\alpha$ and $S$ exists, then $T(\alpha, S):=+\infty$. The minimal time function associated to $S$ for the dynamic $-F$ is denoted by $T(S, \cdot)$. We set

$$
\mathcal{R}_{+}^{S}:=\left\{\alpha \in \mathbb{R}^{\mathrm{n}}: T(S, \alpha)<+\infty\right\},
$$

the set of points attainable (in finite time) by trajectories beginning from $S$. For more information about the minimal time function, see for example $[3,5,20,22]$. We also define the bilateral minimal time function, see $[16,17], T: \mathbb{R}^{\mathrm{n}} \times \mathbb{R}^{\mathrm{n}} \longrightarrow[0,+\infty]$ as follows:

$$
T(\alpha, \beta):=\left\{\begin{array}{l}
\operatorname{Inf} T \geq 0, \\
\dot{x}(t) \in F(x(t)) \text { a.e. } t \in[0, T], \\
x(0)=\alpha \text { and } x(T)=\beta,
\end{array}\right.
$$

(if no trajectory between $\alpha$ from $\beta$ exists, then $T(\alpha, \beta)$ is taken to be $+\infty$ ). We denote by $\mathcal{R}$ the effective domain of $T(\cdot, \cdot)$; that is,

$$
\mathcal{R}:=\left\{(\alpha, \beta) \in \mathbb{R}^{\mathrm{n}} \times \mathbb{R}^{\mathrm{n}}: T(\alpha, \beta)<+\infty\right\} .
$$

In this paper we study the following Hamilton-Jacobi equation:

$$
1+h\left(x, \partial_{P} \varphi(x)\right)=0 \quad \forall x \in \mathcal{R}_{+}^{S}, \varphi(S)=0, \quad\left(\mathrm{HJ}_{S}\right)
$$

where $\partial_{P}$ is the proximal subdifferential. We recall that for a lower semicontinuous function $f: \mathbb{R}^{\mathrm{n}} \longrightarrow \mathbb{R} \cup\{+\infty\}$ and a point $x \in \operatorname{dom} f:=\left\{x^{\prime}: f\left(x^{\prime}\right)<+\infty\right\}, \zeta \in \partial_{P} f(x)$ if and only if there exists $\sigma=\sigma(x, \zeta) \geq 0$ such that

$$
f(y)-f(x)+\sigma\|y-x\|^{2} \geq\langle\zeta, y-x\rangle
$$

for all $y$ in a neighborhood of $x$. A solution of $\left(\mathrm{HJ}_{S}\right)$ means a lower semicontinuous function $\varphi: \mathcal{R}_{+}^{S} \longrightarrow$ $\mathbb{R} \cup\{+\infty\}$ such that $\varphi(S)=0$ and for every $x \in \mathcal{R}_{+}^{S}$, for every $\zeta \in \partial_{P} \varphi(x)$ (if any), we have $h(x, \zeta)+1=0$ (we say that $\varphi(x)$ is a proximal solution, see [8]). This is equivalent to the statement that $\varphi$ is a lower semicontinuous viscosity solution of the following Hamilton-Jacobi equation:

$$
H\left(x,-\varphi^{\prime}(x)\right)-1=0 \quad \forall x \in \mathcal{R}_{+}^{S}, \varphi(S)=0,
$$

where $H$ is the upper Hamiltonian associated to $F$ defined by $H(x, p):=\max _{v \in F(x)}\langle p, v\rangle^{1}$.

It is well-known that the minimal time function $T(\cdot, S)$ is a solution of $\left(\mathrm{HJ}_{S}\right)$ if we replace $\mathcal{R}_{+}^{S}$ by $\mathcal{R}_{+}^{S} \backslash S$ (see for example $[1,3,7,18,22]$ ), but it is never a solution on $\mathcal{R}_{+}^{S}$ since for all $\alpha \in S$ we have $0 \in \partial_{P} T(\cdot, S)(\alpha)$ and $h(\alpha, 0)=0$. In [11], Clarke and Nour study the Hamilton-Jacobi equation $\left(\mathrm{HJ}_{S}\right)$ in the case $S=\left\{\alpha_{0}\right\}$ (we denote this equation by $\left.\left(\mathrm{HJ}_{\alpha_{0}}\right)\right)^{2}$. Let us recall the principal result of [11]. We say that $-F$ is $\alpha_{0}$-STLC $\left(\alpha_{0}\right.$-small-time locally controllable) if and only if the minimal time function $T\left(\alpha_{0}, \cdot\right)$ is continuous at $\alpha_{0}$. This is equivalent to: $\mathcal{R}_{+}^{\alpha_{0}}$ is open, $T\left(\alpha_{0}, \cdot\right)$ is continuous in $\mathcal{R}_{+}^{\alpha_{0}}$ and for any $\beta \in \partial \mathcal{R}_{+}^{\alpha_{0}}$ we have

$$
\lim _{\alpha \longrightarrow \beta} T\left(\alpha_{0}, \alpha\right)=+\infty .
$$

We define $\mathcal{G}_{\alpha_{0}}:=\left\{\Gamma \subset \mathcal{R}_{+}^{\alpha_{0}}:\right.$ there exists a sequence $\beta_{i} \in \Gamma$ such that $\left.T\left(\alpha_{0}, \beta_{i}\right) \longrightarrow+\infty\right\}$. The following theorem is proven in [11] (see [11], Ths. 5.2 and 5.3).

\footnotetext{
${ }^{1}$ See $[4,15,18]$ for the definition of lower semicontinuous viscosity solutions. For an historical references about viscosity solution, see $[12-14]$.

${ }^{2}$ When $S=\{\beta\}$ then in all our notations, we replace $S$ by $\beta$.
} 
Theorem 0.1 (existence of solutions of $\left(\mathrm{HJ}_{\alpha_{0}}\right)$ ). Assume that $-F$ is $\alpha_{0}$-STLC. Then $\mathcal{G}_{\alpha_{0}}$ is nonempty and for $\Gamma \in \mathcal{G}_{\alpha_{0}}$ the function $\varphi_{\Gamma}: \mathcal{R}_{+}^{S} \longrightarrow \mathbb{R} \cup\{-\infty,+\infty\}$ defined by:

$$
\varphi_{\Gamma}(\alpha):=\liminf _{\substack{\alpha^{\prime}-\rightarrow \alpha, \beta \in \Gamma \\ T\left(\alpha_{0}, \beta\right)-\rightarrow+\infty}}\left[T\left(\alpha^{\prime}, \beta\right)-T\left(\alpha_{0}, \beta\right)\right],
$$

is a solution of $\left(\mathrm{HJ}_{\alpha_{0}}\right)$. Moreover, if we denote by $\varphi_{\alpha_{0}}$ the function $\varphi_{\Gamma}$ corresponding to the choice $\mathcal{R}_{+}^{\alpha_{0}}$ of $\Gamma$, then $\varphi_{\alpha_{0}}$ is the minimal solution of $\left(\mathrm{HJ}_{\alpha_{0}}\right)$.

After Theorem 0.1, Clarke and Nour studied in [11] the regularity of solutions and the linear case. They also proved an important relationship between these solutions and global geodesics trajectories. As in [22], the methods used by Clarke and Nour in [11] are based upon nonsmooth proximal monotonicity-invariance considerations developed in [10].

The purpose of this paper is the generalization of the results of [11] for a general compact target set $S$. First, we show by an example (see Ex. 2.7) that if we only assume that $-F$ is $S$-STLC (that is, $T(S, \cdot)$ is continuous) then we cannot guarantee the existence of solutions of $\left(\mathrm{HJ}_{S}\right)$ as in the case $S=\left\{\alpha_{0}\right\}$. Then by adding another hypothesis (which is always satisfied if $S$ is a single point), we show an existence theorem for $\left(\mathrm{HJ}_{S}\right)$ which generalizes Theorem 0.1 . We also prove that this hypothesis is a necessary condition for the existence of solutions and then we generalize all the results of [11]. A number of examples (see Ex. 2.7 in which we prove the existence of solutions of $\left(\mathrm{HJ}_{\alpha_{0}}\right)$ not of the form presented in Th. 0.1$)^{3}$ and some applications concerning the global eikonal equations are also given throughout this paper.

The plan of the paper is as follows. In the next section we present some preliminaries. Several results concerning the existence and the regularity of solutions, and their relations with semigeodesics trajectories are presented in Section 2. In Section 3, we study the minimal solution of $\left(\mathrm{HJ}_{S}\right)$. The results of Sections 2 and 3 are applied to eikonal equations in Section 4. Finally, Section 5 is devoted to the existence of geodesics passing through $S$.

\section{Preliminaries}

\subsection{Notation and hypotheses}

For $\rho>0$ we denote by $B(0 ; \rho):=\left\{x \in \mathbb{R}^{\mathrm{n}}:\|x\|<\rho\right\}$ and $\bar{B}(0 ; \rho):=\left\{x \in \mathbb{R}^{\mathrm{n}}:\|x\| \leq \rho\right\}$. The open (resp. closed) unit ball in $\mathbb{R}^{\mathrm{n}}$ is denoted by $B$ (resp. $\bar{B}$ ). For a set $A \subset \mathbb{R}^{\mathrm{n}}$, int $A, \partial A$ and co $A$ are the interior, the boundary and the convex hull of $A$, respectively.

Definition 1.1. Let $\lambda \in] 0,1]$. We say that:

- $F$ is $S$-LC (S-locally controllable), if $S \subset \operatorname{int} \mathcal{R}_{+}^{S}$.

- $F$ is $S$-STLC ( $S$-small-time locally controllable), if $T(\cdot, S)$ is continuous at every point of $\partial S$.

- $F$ satisfies the hypothesis $\left(H_{\lambda}\right)$ at $\beta \in \mathbb{R}^{\mathrm{n}}$, if there exist $r>0$ and $\delta>0$ such that for any $\beta^{\prime} \in B(\beta ; r)$ and for any unit vector $\gamma$ we have

$$
h\left(\beta^{\prime}, \gamma\right)<\frac{-\delta\left\|\beta^{\prime}-\beta\right\|^{1-\lambda}}{\lambda}{ }^{4}
$$

We denote by $\mathcal{R}_{-}^{S}:=\left\{\alpha \in \mathbb{R}^{\mathrm{n}}: T(\alpha, S)<+\infty\right\}$, the set of points which can be steered to $S$ in finite time. It is well-known (see $[3,17,19])$ that:

- $F$ is $S$-LC $\Longleftrightarrow \mathcal{R}_{-}^{S}$ is open.

\footnotetext{
3 This is an important question not treated in [11].

4 The hypothesis $\left(H_{\lambda}\right)$ is known by the Petrov $\lambda$-Hölder modulus condition.
} 
- $F$ is $S$-STLC $\Longleftrightarrow \mathcal{R}_{-}^{S}$ is open, $T(\cdot, S)$ is continuous in $\mathcal{R}_{-}^{S}$ and for any $\beta \in \partial \mathcal{R}_{-}^{S}$ we have

$$
\lim _{\alpha \longrightarrow \beta} T(\alpha, S)=+\infty .
$$

- For $\beta \in \mathbb{R}^{\mathrm{n}}$ we have:

- $F$ satisfies $\left(H_{\lambda}\right)$ at $\left.\left.\beta(\lambda \in] 0,1\right]\right) \Longrightarrow F$ is $\beta$-STLC.

- $F$ satisfies $\left(H_{1}\right)$ at $\beta \Longleftrightarrow 0 \in \operatorname{int} F(\beta) \Longrightarrow F$ is $\beta$-STLC.

The basic hypotheses in force throughout the article are the following:

- $S$ is a nonempty compact set.

- $\operatorname{int} S=\emptyset$.

- $-F$ is $S$-LC (which is equivalent to $\mathcal{R}_{+}^{S}$ is open).

We note that we must assume that int $S=\emptyset$. This assumption is related to the nature of the semicontinuous solution chosen here. Indeed, if int $S=\emptyset$, then a solution $\varphi$ of $\left(\mathrm{HJ}_{S}\right)$ vanishes on int $S$ and then $0 \in \partial_{P} \varphi(x)$ for all $x \in \operatorname{int} S$, which gives a contradiction since $h(\cdot, 0)=0$.

\subsection{Monotonicity of trajectories}

Let $\Omega$ be an open subset of $\mathbb{R}^{\mathrm{n}}$ and let $\varphi: \mathbb{R}^{\mathrm{n}} \longrightarrow \mathbb{R} \cup\{+\infty\}$ be an extended real-valued function which is lower semicontinuous on $\Omega$ with $\operatorname{dom} \varphi \cap \Omega \neq \emptyset$. We say that the system $(\varphi, F)$ is strongly increasing on $\Omega$ if for any trajectory $x$ on an interval $[a, b]$ for which $x([a, b]) \subset \Omega$, we have

$$
\varphi(x(s)) \leq \varphi(x(t)) \forall s, t \in[a, b], s \leq t .
$$

The system $(\varphi, F)$ is said to be weakly decreasing on $\Omega$ if for every $\alpha \in \Omega$ there is a trajectory $x$ on a nontrivial interval $[a, b]$ satisfying

$$
x(a)=\alpha, \varphi(x(t)) \leq \varphi(\alpha) \quad \forall t \in[a, b] ;
$$

by reducing $b$ if necessary we may also arrange to have $x([a, b]) \subset \Omega$. In each case, one obtains an equivalent definition by requiring the inequality to hold on $[a, \tau[$, where $\tau \in] a,+\infty]$ is the exit time of the trajectory $x$ from $\Omega$ : the supremum of all $T>0$ having the property that $x([a, T]) \subset \Omega$. The following proposition is proven in [10], Chapter 4, Section 6. See also [2] and [9].

Proposition 1.2. The system $(\varphi, F)$ is strongly increasing on $\Omega$ if and only if

$$
h\left(x, \partial_{P} \varphi(x)\right) \geq 0 \quad \forall x \in \Omega,
$$

and weakly decreasing on $\Omega$ if and only if

$$
h\left(x, \partial_{P} \varphi(x)\right) \leq 0 \quad \forall x \in \Omega .
$$

\section{EXISTENCE OF SOLUTIONS}

We begin this section by the following proposition which gives some properties of a solution of $\left(\mathrm{HJ}_{S}\right)$. The proof follows using Proposition 1.2. The details is left to the reader (see the proof of [11], Prop. 4.4).

Proposition 2.1. Let $\varphi$ a solution of $\left(\mathrm{HJ}_{S}\right)$. Then we have:

(i) $T(\alpha, \beta)+\varphi(\beta) \geq \varphi(\alpha)$, for all $\alpha, \beta \in \mathcal{R}_{+}^{S}$.

(ii) $T(\alpha, S) \geq \varphi(\alpha) \geq-T(S, \alpha)$, for all $\alpha \in \mathcal{R}_{+}^{S}$ (and then $\mathcal{R}_{+}^{S} \cap \mathcal{R}_{-}^{S} \subset \operatorname{dom} \varphi$ ).

(iii) For every $\alpha \in \operatorname{dom} \varphi$ there exists a trajectory $x$ of $F$ such that $x(0)=\alpha$ and

$$
\varphi(x(t))+t=\varphi(\alpha) \quad \forall t \geq 0 .
$$


Now let $\alpha \in \mathbb{R}^{\mathrm{n}}$. A trajectory $x:\left[0,+\infty\left[\longrightarrow \mathbb{R}^{\mathrm{n}}\right.\right.$ of $F$ is a semigeodesic from $\alpha$ if and only if $x(0)=\alpha$ and $T(x(s), x(t))=t-s$ for all $s \leq t \in[0,+\infty[$. If $\alpha \in S$, then we say that $x$ is a semigeodesic from $S$. The following proposition presents the relationship between a solution of $\left(\mathrm{HJ}_{S}\right)$ and semigeodesic trajectories.

Proposition 2.2. Let $\varphi$ be a solution of $\left(\mathrm{HJ}_{S}\right)$. Then for every $\alpha \in \operatorname{dom} \varphi$ there exists a semigeodesic $x$ from $\alpha$ such that

$$
\varphi(x(t))+t=\varphi(\alpha) \quad \forall t \geq 0 .
$$

Moreover, if $\alpha \in S$ then we have $T(S, x(t))=t \forall t \geq 0$.

Proof. Let $\varphi$ be a solution of $\left(\mathrm{HJ}_{S}\right)$ and let $\alpha \in \operatorname{dom} \varphi$. By Proposition 2.1, there exists a trajectory $x$ : $\left[0,+\infty\left[\longrightarrow \mathbb{R}^{\mathrm{n}}\right.\right.$ of $F$ in $\mathcal{R}_{+}^{S}$ such that $x(0)=\alpha$ and

$$
\varphi(x(t))+t=0 \quad \forall t \geq 0 .
$$

We claim that $x$ is a semigeodesic from $\alpha$. Indeed, let $s \leq t \in[0,+\infty[$, then by (1) and Proposition 2.1 we have

$$
T(x(s), x(t)) \geq \varphi(x(s))-\varphi(x(t))=t-s,
$$

but

$$
T(x(s), x(t)) \leq t-s,
$$

therefore $T(x(s), x(t))=t-s$.

Now we assume that $\alpha \in S$. Then by (1) and Proposition 2.1 we get that

$$
t=T(\alpha, x(t)) \geq T(S, x(t)) \geq-\varphi(x(t))=t \quad \forall t \geq 0 .
$$

Hence $T(S, x(t))=t \forall t \geq 0$.

As mentioned in the introduction, Clarke and Nour proved in [11] that if $S$ is a single point, then under the hypothesis " $-F$ is $S$-STLC" the equation $\left(\mathrm{HJ}_{S}\right)$ always admits a solutions. In the following example we prove, using Proposition 2.2, that this fails in general if $S$ is not a single point. Thus to prove the existence of solutions we must add another hypothesis.

Example 2.3. We consider the same data of [16], Example 7.8; that is for $n=2$, we define $F$ as the following:

- If $\|(x, y)\| \geq 2$, then $F(x, y):=F_{1}(x, y)$ where

$$
F_{1}(x, y):= \begin{cases}\left\{\left(\frac{x^{2}-y^{2}}{x^{2}+y^{2}}, \frac{2 x y}{x^{2}+y^{2}}\right)\right\} & \text { if } y \neq 0, \\ \{(1,0)\} & \text { if } y=0 .\end{cases}
$$

- If $\|(x, y)\| \leq 1$, then $F(x, y):=F_{2}(x, y)$ where $F_{2}(x, y):=\bar{B}$ for all $(x, y) \in \mathbb{R}^{2}$.

- If $1<\|(x, y)\|:=r<2$, then

$$
F(x, y):=\left\{(2-r) v_{2}+(r-1) v_{1}: v_{1} \in F_{1}(x, y) \text { and } v_{2} \in F_{2}(x, y)\right\} .
$$

We take $S:=\left[-\frac{1}{2}, 0\right] \times\{0\}$. Then we can easily verify that all our hypotheses are satisfied and that $-F$ is $S$-STLC. Moreover, we have the following ${ }^{5}$ :

- $\left.\left.\mathcal{R}_{+}^{S}=\mathbb{R}^{2} \backslash\right]-\infty,-2\right] \times\{0\}$.

- For all $(a, 0) \in S$ there exists only one semigeodesic $x:\left[0,+\infty\left[\longrightarrow \mathbb{R}^{2}\right.\right.$ from $(a, 0)$ which is the following trajectory: $x(t)=(t+a, 0)$.

\footnotetext{
5 The proof of these claims follows using the same ideas as in [11], Example 7.8.
} 
Now we assume that the Hamilton-Jacobi equation $\left(\mathrm{HJ}_{S}\right)$ admits a solution $\varphi$; we shall derive a contradiction. By Proposition 2.2 there exists a semigeodesic (it must be the trajectory $x$ defined above) from $\left(-\frac{1}{2}, 0\right)$ such that

But this contradicts the fact that

$$
T(S, x(t))=t \quad \forall t \geq 0 .
$$

$$
x(t) \in S \quad \forall t \in\left[0, \frac{1}{2}\right] .
$$

Now we define the following set of subsets of $\mathcal{R}_{+}^{S}$ :

$\mathcal{G}_{S}:=\left\{\Gamma \subset \mathcal{R}_{+}^{S}:\right.$ for all $\alpha \in S$ there exists a sequence $\beta_{i} \in \Gamma$ such that $\left.T\left(\alpha, \beta_{i}\right)=T\left(S, \beta_{i}\right) \longrightarrow+\infty\right\}$.

Then we have the following theorem which generalizes Theorem 0.1 .

Theorem 2.4 (existence of solutions of $\left.\left(\mathrm{HJ}_{S}\right)\right)$. Assume that $-F$ is $S$-STLC and that $\mathcal{G}_{S}$ is nonempty. Then for $\Gamma \in \mathcal{G}_{S}$ the function $\varphi_{\Gamma}: \mathcal{R}_{+}^{S} \longrightarrow \mathbb{R} \cup\{-\infty,+\infty\}$ defined by:

$$
\varphi_{\Gamma}(\alpha):=\liminf _{\substack{\alpha^{\prime} \rightarrow \alpha, \beta \in \Gamma \\ T(S, \beta) \rightarrow+\infty}}\left[T\left(\alpha^{\prime}, \beta\right)-T(S, \beta)\right]
$$

is a solution of $\left(\mathrm{HJ}_{S}\right)$. Moreover, if we denote by $\varphi_{S}$ the function $\varphi_{\Gamma}$ corresponding to the choice $\mathcal{R}_{+}^{S}$ of $\Gamma$, then $\varphi_{S}$ is the minimal solution of $\left(\mathrm{HJ}_{S}\right)$.

Proof. We proceed exactly as in the proofs of [11], Theorems 5.2 and 5.3. The only difference here is that we must use the definition of $\Gamma \in \mathcal{G}_{S}$ to prove that $\varphi_{\Gamma}(S) \leq 0$.

Remark 2.5. If $S=\left\{\alpha_{0}\right\}$ and $-F$ is $\alpha_{0}$-STLC then the hypothesis " $\mathcal{G}_{S}$ is nonempty" is always satisfied since in this case we have that $T(S, \cdot)=T\left(\alpha_{0}, \cdot\right)$.

In the following corollary we prove that the hypothesis " $\mathcal{G}_{S}$ is nonempty" is also a necessary condition for the existence of solutions.

Corollary 2.6. Assume that $-F$ is $S$-STLC. Then the following statements are equivalent:

(i) The Hamilton-Jacobi equation $\left(\mathrm{HJ}_{S}\right)$ admits a solution.

(ii) For every $\alpha \in S$ there exists a semigeodesic $x$ from $\alpha$ such that $T(S, x(t))=t \forall t \geq 0$.

(iii) The set $\mathcal{G}_{S}$ is nonempty.

(iv) The Hamilton-Jacobi equation $\left(\mathrm{HJ}_{S}\right)$ admits a minimal solution.

Proof. Follows from Proposition 2.2 and Theorem 2.4.

Now we give an example in which we prove, using Theorem 2.4, that $\left(\mathrm{HJ}_{S}\right)$ may admit a solution not of the form $\varphi_{\Gamma}$ presented in Theorem 0.1 .

Example 2.7. For $n=1$, let $F(x)=-x+[-1,1]$. It is easy to prove that $\mathcal{R}=\mathcal{R}_{1} \cup \mathcal{R}_{2}$ where

- $\mathcal{R}_{1}=\{(x, y) \in \mathbb{R} \times \mathbb{R}:-1<y \leq x\}$;

- $\mathcal{R}_{2}=\{(x, y) \in \mathbb{R} \times \mathbb{R}: x \leq y<1\}$.

We calculate $T(\cdot, \cdot)$ and we find that:

$$
T(x, y)= \begin{cases}\ln \left(\frac{1+x}{1+y}\right) & \text { if }(x, y) \in \mathcal{R}_{1} \\ \ln \left(\frac{1-x}{1-y}\right) & \text { if }(x, y) \in \mathcal{R}_{2}\end{cases}
$$

In this example we have $h(x, p)=-\langle x, p\rangle-\|p\|$ and then the Hamilton-Jacobi equation $\left(\mathrm{HJ}_{S}\right)$ is

$$
\left\langle x, \partial_{P} \varphi(x)\right\rangle+\left\|\partial_{P} \varphi(x)\right\|=1 \quad \forall x \in \mathbb{R}, \quad \varphi(S)=0 .
$$


We consider four cases of $S$ (in all these cases, we have that $-F$ is $S$-STLC):

- $S_{1}=\left\{\frac{1}{2}\right\}$ :

Clearly we have $\left.\mathcal{R}_{+}^{\frac{1}{2}}=\right]-1,1\left[\right.$. Moreover, there exist only two semigeodesics from $\frac{1}{2}$ which are:

(i) $x(t)=1-\frac{1}{2} \mathrm{e}^{-t}, t \geq 0$.

(ii) $y(t)=\frac{3}{2} \mathrm{e}^{-t}-1, t \geq 0$.

Let us calculate all the solutions of $\left(\mathrm{HJ}_{\frac{1}{2}}\right)$ of the form $\varphi_{\Gamma}$. Let $\Gamma \in \mathcal{G}_{\frac{1}{2}}$, then there exist three cases:

(a) $\Gamma$ contains a sequence which converges to 1 and does not contain a sequence which converges to -1 . Then we find the following solution of $\left(\mathrm{HJ}_{\frac{1}{2}}\right)$ :

$$
\varphi_{\Gamma_{1}}(x)=\ln (2)+\ln (1-x) .
$$

(b) $\Gamma$ contains a sequence which converges to -1 and does not contain a sequence which converges to 1 . Then we find the following solution of $\left(\mathrm{HJ}_{\frac{1}{2}}\right)$ :

$$
\varphi_{\Gamma_{2}}(x)=-\ln \left(\frac{3}{2}\right)+\ln (1+x)
$$

(c) $\Gamma$ contains a sequence which converges to -1 and a sequence which converges to 1 . Then we find the minimal solution of $\left(\mathrm{HJ}_{\frac{1}{2}}\right)$ :

$$
\varphi_{\frac{1}{2}}(x)=-T\left(\frac{1}{2}, x\right)= \begin{cases}\ln (2)+\ln (1-x) & \text { if } x \in\left[\frac{1}{2}, 1[\right. \\ -\ln \left(\frac{3}{2}\right)+\ln (1+x) & \text { if } \left.x \in]-1, \frac{1}{2}\right] .\end{cases}
$$

- $S_{2}=\left\{-\frac{1}{2}\right\}$ :

As in the preceding case we have $\left.\mathcal{R}_{+}^{-\frac{1}{2}}=\right]-1,1\left[\right.$ and there exist only two semigeodesics from $-\frac{1}{2}$ which are:
(i) $x^{\prime}(t)=1-\frac{3}{2} \mathrm{e}^{-t}, t \geq 0$.
(ii) $y^{\prime}(t)=\frac{1}{2} \mathrm{e}^{-t}-1, t \geq 0$.

The solutions of $\left(\mathrm{HJ}_{-\frac{1}{2}}\right)$ of the form $\varphi_{\Gamma}$ are the following:

(a) $\varphi_{\Gamma_{3}}(x)=\ln (1-x)-\ln \left(\frac{3}{2}\right)$.

(b) $\varphi_{\Gamma_{4}}(x)=\ln (2)+\ln (1+x)$.

(c) $\varphi_{-\frac{1}{2}}(x)=-T\left(-\frac{1}{2}, x\right)= \begin{cases}-\ln \left(\frac{3}{2}\right)-\ln (1-x) & \text { if } x \in\left[-\frac{1}{2}, 1[\right. \\ \ln (2)+\ln (1+x) & \left.\text { if } x \in]-1,-\frac{1}{2}\right] .\end{cases}$

- $S_{3}=\left\{-\frac{1}{2}, \frac{1}{2}\right\}$ :

Clearly we have $\left.\mathcal{R}_{+}^{S_{3}}=\right]-1,1\left[\right.$. We claim that $\mathcal{G}_{S_{3}} \neq \emptyset$. Indeed, for $\alpha=\frac{1}{2}$ the semigeodesic $x(t)=$ $1-\frac{1}{2} \mathrm{e}^{-t}$ satisfies $T\left(S_{3}, x(t)\right)=t \forall t \geq 0$, and for $\alpha=-\frac{1}{2}$ the semigeodesic $y^{\prime}(t)=\frac{1}{2} \mathrm{e}^{-t}-1$ satisfies $T\left(S_{3}, y^{\prime}(t)\right)=t \forall t \geq 0$. Then by Theorem 2.4, the Hamilton-Jacobi equation $\left(\mathrm{HJ}_{S_{3}}\right)$ admits a minimal 
solution $\varphi_{S_{3}}$. Using Theorem 2.4, we calculate this solution and we find that

$$
\varphi_{S_{3}}(x)= \begin{cases}\ln (2)+\ln (1-x) & \text { if } x \in[0,1[ \\ \ln (2)+\ln (1+x) & \text { if } x \in]-1,0]\end{cases}
$$

But $\varphi_{S_{3}}$ is also a solution of $\left(\mathrm{HJ}_{\frac{1}{2}}\right)$, then $\left(\mathrm{HJ}_{\frac{1}{2}}\right)$ admits a solution not of the form $\varphi_{\Gamma}$ (all the solutions of the form $\varphi_{\Gamma}$ calculated above are different of $\left.\varphi_{S_{3}}\right)$.

- $S_{4}=\left\{-\frac{1}{2}, 0, \frac{1}{2}\right\}$ :

In this case we also have that $\left.\mathcal{R}_{+}^{S_{4}}=\right]-1,1\left[\right.$. We claim that $\mathcal{G}_{S_{4}}=\emptyset$ (and then $\left(\mathrm{HJ}_{S_{4}}\right.$ ) does not admit any solution). Indeed, we cannot find any semigeodesic $z$ from 0 which satisfies $T\left(S_{4}, z(t)\right)=t$ for all $t \geq 0$.

The following proposition presents how we can calculate a solution of $\left(\mathrm{HJ}_{S}\right)$ using the solutions of $\left(\mathrm{HJ} \mathrm{J}_{\alpha}\right)$ for all $\alpha \in S$.

Proposition 2.8. We have the following statements:

(a) Let $\alpha \in S$ and assume that $S \subset \mathcal{R}_{+}^{\alpha}$ and that $-F$ is $\alpha$-LC. We consider the following statements:

(i) There exists a solution $\psi_{\alpha}$ of $\left(\mathrm{HJ}_{\alpha}\right)$ such that $\psi_{\alpha}(S) \geq 0$.

(ii) There exists a semigeodesic $x$ from $\alpha$ such that $T(S, x(t))=t \forall t \geq 0$.

Then $(i) \Longrightarrow($ ii $)$ and the reverse implication is true if we add that $-F$ is $\beta$-STLC for all $\beta \in S$.

(b) Assume that $S \times S \subset \mathcal{R}$ and that $-F$ is $S$-STLC. Assume further that for all $\alpha \in S$ there exists a solution $\psi_{\alpha}$ of $\left(\mathrm{HJ}_{\alpha}\right)$ such that $\psi_{\alpha}(S) \geq 0$. Then $\mathcal{G}$ is a nonempty set. Moreover, the function $\bar{\varphi}: \mathcal{R}_{+}^{S} \longrightarrow$ $\mathbb{R} \cup\{-\infty,+\infty\}$ defined by

$$
\bar{\varphi}(x):=\liminf _{x^{\prime} \longrightarrow x} \varphi\left(x^{\prime}\right), \text { where } \varphi(x):=\inf _{\alpha \in S} \varphi_{\alpha}(x),
$$

is a solution of $\left(\mathrm{HJ}_{S}\right)$.

Proof. $(a)(i) \Longrightarrow($ ii): We apply Proposition 2.2 (for $S=\{\alpha\}$ ) and we get that for all $\alpha \in S$ there exists a semigeodesic $x_{\alpha}$ from $\alpha$ such that

$$
\psi_{\alpha}\left(x_{\alpha}(t)\right)+t=0 \quad \forall t \geq 0 .
$$

We claim that $T\left(S, x_{\alpha}(t)\right)=t$ for all $t \geq 0$. Indeed, let $\beta \in S$, then by Proposition 2.1 we have

$$
T\left(\beta, x_{\alpha}(t)\right)+\psi_{\alpha}\left(x_{\alpha}(t)\right) \geq \psi_{\alpha}(\beta) \geq 0 \quad \forall t \geq 0 .
$$

Hence

$$
T\left(\beta, x_{\alpha}(t)\right) \geq-\psi_{\alpha}\left(x_{\alpha}(t)\right)=t \quad \forall t \geq 0,
$$

which gives ( since $T\left(\alpha, x_{\alpha}(t)\right)=t$ for all $t \geq 0$ ) that $T\left(S, x_{\alpha}(t)\right)=t$ for all $t \geq 0$.

Now we assume further that $-F$ is $\beta$-STLC for all $\beta \in S$. Let us prove that $(i i) \Longrightarrow(i)$. We consider $\Gamma:=\{x(t): t \geq 0\}$ and we denote by $\psi_{\alpha}$ the solution of $\left(\mathrm{HJ}_{\alpha}\right)$ corresponding to $\Gamma$. Let $\beta \in S$. Then we have

$$
0 \leq T(\beta, x(t))-T(\alpha, x(t)) \leq T\left(\beta, \beta^{\prime}\right)+T\left(\beta^{\prime}, x(t)\right)-T(\alpha, x(t)) \quad \forall \beta^{\prime} \in \mathcal{R}_{+}^{\alpha},
$$

hence

$$
T\left(\beta^{\prime}, x(t)\right)-T(\alpha, x(t)) \geq-T\left(\beta, \beta^{\prime}\right) \quad \forall \beta^{\prime} \in \mathcal{R}_{+}^{\alpha},
$$

which gives by the definition of $\psi_{\alpha}$ and by the continuity of $T(\beta, \cdot)$ that $\psi_{\alpha}(\beta) \geq 0$. 
(b) Since $S \times S \in \mathcal{R}$ we have that the open set $\mathcal{R}_{+}^{S}=\mathcal{R}_{+}^{\alpha}$ for all $\alpha \in S$. Then by $(a)$ we get that $\mathcal{G} \neq \emptyset$. Now let $\alpha \in S$ and let $\beta \in \mathcal{R}_{+}^{S}$. We consider $\theta \in S$ such that $T(\theta, \beta)=T(S, \beta)$. By Proposition 2.1 (applied for $S=\{\alpha\})$ we have

$$
T(S, \beta)+\psi_{\alpha}(\beta)=T(\theta, \beta)+\psi_{\alpha}(\beta) \geq \psi_{\alpha}(\theta) \geq 0,
$$

then

$$
\psi_{\alpha}(\beta) \geq-T(S, \beta)
$$

Hence for all $\alpha \in S$ we have $\psi_{\alpha}(\cdot) \geq-T(S, \cdot)$ which gives (by the continuity of $T(S, \cdot)$ ) that

$$
-T(S, \cdot) \geq \bar{\varphi}(\cdot) \geq \varphi(\cdot) .
$$

Therefore $\bar{\varphi}(\cdot)>-\infty$ and it is lower semicontinuous. Moreover, $\bar{\varphi}(S)=0$ since for all $\beta \in S$ we have

$$
0=-T(S, \beta) \geq \bar{\varphi}(\beta) \geq \varphi(\beta)=\inf _{\alpha \in S} \varphi_{\alpha}(\beta)=0 .
$$

Now we prove that $1+h\left(x, \partial_{P} \bar{\varphi}(x)\right)=0$ for all $x \in \mathcal{R}_{+}^{S}$. It is sufficient to prove that $t+\bar{\varphi}$ is weakly decreasing and strongly increasing. We begin by strongly increasing.

Let $x:[a, b] \longrightarrow \mathbb{R}^{\mathrm{n}}$ be a trajectory of $F$ such that $x([a, b]) \subset \mathcal{R}_{+}^{S}$ and let $t \in[a, b]$. We need to prove that

$$
t+\bar{\varphi}(x(t)) \leq b+\bar{\varphi}(b) .
$$

We can assume that $x(b) \in \operatorname{dom} \bar{\varphi}$. Then there exists a sequence $\beta_{i} \longrightarrow \beta$ such that

$$
\bar{\varphi}(x(b))=\lim _{i \longrightarrow+\infty} \varphi\left(\beta_{i}\right) .
$$

By the continuous dependence on the initial condition (see [10], Th. 4.3.11) there exists a sequence of trajectories $x_{i}$ on $[t, b]$ such that $x_{i}(b)=\beta_{i}$ and

$$
\lim _{i \rightarrow+\infty} x_{i}(t)=x(t)
$$

Clearly the trajectory $x_{i}$ remains in $\mathcal{R}_{+}^{S}$ (since it begins in $\mathcal{R}_{+}^{S}$ ), then since $\psi_{\alpha}$ is a solution of $\left(\mathrm{HJ}_{\alpha}\right)$ for all $\alpha \in S$, we have

Hence

$$
t+\psi_{\alpha}\left(x_{i}(t)\right) \leq b+\psi_{\alpha}\left(x_{i}(b)\right) \quad \forall \alpha \in S
$$

Taking $i \longrightarrow+\infty$ we get

$$
t+\varphi\left(x_{i}(t)\right) \leq b+\varphi\left(x_{i}(b)\right) \leq b+\bar{\varphi}\left(x_{i}(b)\right)
$$

The strong increase follows.

$$
t+\bar{\varphi}(x(t)) \leq b+\bar{\varphi}(x(b))
$$

Now we prove the weak decrease property. Let $\beta \in \operatorname{dom} \bar{\varphi}$, then there exists a sequence $\beta_{i} \longrightarrow \beta$ such that

$$
\bar{\varphi}(x(b))=\lim _{i \longrightarrow+\infty} \varphi\left(\beta_{i}\right) .
$$

By the definition of $\varphi$ we have that for all $i$ there exists $\alpha_{i} \in S$ such that

$$
\psi_{\alpha_{i}}\left(\beta_{i}\right) \leq \varphi\left(\beta_{i}\right)+\frac{1}{i}
$$

Since $\psi_{\alpha_{i}}$ is a solution of $\left(\mathrm{HJ}_{\alpha_{i}}\right)$ for all $i$, there exists a sequence of trajectories $x_{i}$ on $\left[0,+\infty\left[\right.\right.$ such that $x_{i}(0)=\beta_{i}$ and

$$
\psi_{\alpha_{i}}\left(\beta_{i}\right) \geq t+\psi_{\alpha_{i}}\left(x_{i}(t)\right) \quad \forall t \geq 0 .
$$


By the compactness property of trajectories, we can assume that there exists a trajectory $x$ on $[0,+\infty[$ such that $x(0)=\beta$ and $x_{i} \longrightarrow x$ uniformly on compact interval. By (2) and (3) we have

$$
\varphi\left(\beta_{i}\right)+\frac{1}{i} \geq \psi_{\alpha_{i}}\left(\beta_{i}\right) \geq t+\psi_{\alpha_{i}}\left(x_{i}(t)\right) \geq t+\varphi\left(x_{i}(t)\right) \quad \forall t \geq 0 .
$$

Taking $i \longrightarrow+\infty$ we get

The weak decrease follows.

$$
\bar{\varphi}(\beta) \geq t+\bar{\varphi}(x(t)) \quad \forall t \geq 0 .
$$

Example 2.9. We return to Example 2.7 and we remark that

- $\varphi_{\Gamma_{1}}\left(-\frac{1}{2}\right)=\ln (3)>0$,

- $\varphi_{\Gamma_{4}}\left(\frac{1}{2}\right)=\ln (3)>0$.

Then by Proposition 2.8 (clearly for $S_{3}=\left\{-\frac{1}{2}, \frac{1}{2}\right\}$ we have $S_{3} \times S_{3} \subset \mathcal{R}$ ) we get that the function $\min \left\{\varphi_{\Gamma_{1}}, \varphi_{\Gamma_{4}}\right\}$ is a solution of $\left(\mathrm{HJ}_{S_{3}}\right)$. If we calculate this function we find exactly the minimal solution $\varphi_{S_{3}}$.

Now we study the regularity of solutions.

Proposition 2.10. Let $\varphi$ be a solution of $\left(\mathrm{HJ}_{S}\right)$ and let $\alpha \in \operatorname{dom} \varphi$. Then we have:

(i) $F$ is $\alpha$-STLC $\Longrightarrow \varphi$ is continuous at $\alpha$.

(ii) F satisfies $\left(H_{\lambda}\right)$ at $\left.\left.\alpha(\lambda \in] 0,1\right]\right) \Longrightarrow \varphi$ is $\lambda$-Hölder continuous near $\alpha .^{6}$

Proof.

(i) Follows exactly as the proof of (1) of [11], Proposition 6.1.

(ii) We proceed as in the proof of (2) of [11], Proposition 6.1, and we use [17], Proposition 4.4, which asserts the existence of $\rho>0$ such that $T(\cdot, \cdot)$ is $\lambda$-Hölder continuous in $B(\alpha, \rho) \times B(\alpha, \rho)$.

Proposition 2.11. We have the following statements:

(i) Assume that $F$ is $\beta$-STLC for all $\beta \in \mathcal{R}_{+}^{S} \cap \mathcal{R}_{-}^{S}$. Then all solutions of $\left(\mathrm{HJ}_{S}\right)$ are continuous in the open set $\mathcal{R}_{+}^{S} \cap \mathcal{R}_{-}^{S}$.

(ii) Assume that $F$ satisfies $\left(H_{\lambda}\right)$ at $\beta$ for all $\left.\left.\beta \in \mathcal{R}_{+}^{S} \cap \mathcal{R}_{-}^{S}((\lambda \in] 0,1]\right)\right)$. Then all solutions of $\left(\mathrm{HJ}_{S}\right)$ are locally $\lambda$-Hölder continuous in the open set $\mathcal{R}_{+}^{S} \cap \mathcal{R}_{-}^{S}$.

Proof. Since $S \subset \mathcal{R}_{+}^{S} \cap \mathcal{R}_{-}^{S}$, we have that $F$ is $S$-STLC in 1) and 2). Then $\mathcal{R}_{-}^{S}$ is open and hence $\mathcal{R}_{+}^{S} \cap \mathcal{R}_{-}^{S}$ is open. By Proposition 2.1 we have $\mathcal{R}_{+}^{S} \cap \mathcal{R}_{-}^{S} \subset \operatorname{dom} \varphi$ for all $\varphi$ a solution of $\left(\mathrm{HJ}_{S}\right)$. Then by Proposition 2.10 we find the two results.

\section{The Minimal SOlUtion}

In this section we present the relationship between the minimal solution $\varphi_{S}$ and the function $-T(S, \cdot)$. The semigeodesics $x$ from $S$ which satisfies $T(S, x(t))=t$ for all $t \geq 0$, will play an important role in this study. We note that in all this section, we assume that $-F$ is $S$-STLC and that $\mathcal{G}_{S} \neq \emptyset$.

Theorem 3.1. Let $x:\left[0,+\infty\left[\longrightarrow \mathbb{R}^{\mathrm{n}}\right.\right.$ be a trajectory of $F$ from $S$. Then the following statements are equivalent:

(i) The trajectory $x$ is a semigeodesic from $S$ which satisfies $T(S, x(t))=t$ for all $t \geq 0$.

(ii) For all $t \geq 0$, we have $\varphi_{S}(x(t))+t=0$.

\footnotetext{
${ }^{6}$ If $\lambda=1$ then we obtain that $0 \in \operatorname{int} F(\alpha) \Longrightarrow \varphi$ is Lipschitz near $\alpha$.
} 
Proof. To prove the implication $(i) \Longrightarrow($ ii $)$ and the first part of the implication $(i i) \Longrightarrow(i)(x$ is a semigeodesic from $S$ ) we proceed exactly as in the proof of [11], Theorem 7.4. Let us prove the second part of $(i i) \Longrightarrow(i)$, that is, $T(S, x(t))=t$ for all $t \geq 0$. Let $\alpha \in S$, then by Proposition 2.1 we have

$$
T(\alpha, x(t))+\varphi_{S}(x(t)) \geq \varphi_{S}(\alpha)=0 \quad \forall t \geq 0
$$

hence

$$
T(\alpha, x(t)) \geq-\varphi_{S}(x(t))=t \quad \forall t \geq 0 .
$$

This gives that

$$
t \leq T(S, x(t)) \leq T(x(0), x(t))=t \quad \forall t \geq 0 .
$$

The proof is completed.

The following theorem gives a necessary and sufficient condition for $\varphi_{S}(\alpha)$ to be $-T(S, \alpha)$.

Theorem 3.2. Let $\alpha \in \mathcal{R}_{+}^{S}$. Then the following statements are equivalent:

(i) The point $\alpha$ lies on a semigeodesic from $S$ which satisfies $T(S, x(t))=t$ for all $t \geq 0$.

(ii) $\varphi_{S}(\alpha)=-T(S, \alpha)$.

Proof. See the proof of [11], Theorem 7.6.

Remark 3.3. In Example 2.7 if we calculate the function $-T\left(S_{3}, \cdot\right)\left(S_{3}=\left\{-\frac{1}{2}, \frac{1}{2}\right\}\right)$ then we obtain the following:

$$
-T\left(S_{3}, x\right)= \begin{cases}\ln (2)+\ln (1-x) & \text { if } x \in\left[\frac{1}{2}, 1\right], \\ -\ln \left(\frac{3}{2}\right)-\ln (1+x) & \text { if } x \in\left[0, \frac{1}{2}\right], \\ -\ln \left(\frac{3}{2}\right)+\ln (1-x) & \text { if } x \in\left[-\frac{1}{2}, 0\right], \\ \ln (2)+\ln (1+x) & \text { if } \left.x \in]-1,-\frac{1}{2}\right] .\end{cases}
$$

We remark that $\varphi_{S_{3}}(\cdot)$ and $-T\left(S_{3}, \cdot\right)$ coincide only on $\left.]-1,-\frac{1}{2}\right] \cup\left[\frac{1}{2}, 1[\right.$. We can easily deduce this from Theorem 3.2. Indeed, for all $\alpha \in$ ] $-\frac{1}{2}, \frac{1}{2}$ [ we can not find any semigeodesic from $S_{3}$ passing through $\alpha$ and which satisfies $T\left(S_{3}, x(t)\right)=t$ for all $t \geq 0$, but if we take $\alpha \in\left[\frac{1}{2}, 1[\right.$ (resp. $\left.\alpha \in]-1,-\frac{1}{2}\right]$ ) then the semigeodesic $x(t)=1-\frac{1}{2} \mathrm{e}^{-t}\left(\right.$ resp. $\left.x(t)=\frac{1}{2} \mathrm{e}^{-t}-1\right)$ begins from $S_{3}$, passes through $\alpha$ and satisfies $T\left(S_{3}, x(t)\right)=t$ for all $t \geq 0$. We also note that in this example we have $\varphi_{\frac{1}{2}}(\cdot)=-T\left(\frac{1}{2}, \cdot\right)$ and $\varphi_{-\frac{1}{2}}(\cdot)=-T\left(-\frac{1}{2}, \cdot\right)$ but $\varphi_{S_{3}}(\cdot) \neq-T\left(S_{3}, \cdot\right)$.

We recall (see [11]) that a continuous function $\varphi$ is said to be mildly regular at a point $x$ if it satisfies $\partial^{P} \varphi(x) \subset \partial_{L} \varphi(x)$, where $\partial^{P} \varphi(x)$ is the proximal superdifferential of $\varphi$ at $x$ defined (for an upper semicontinuous function) by $\partial^{P} \varphi(x):=-\partial_{P}(-\varphi)(x)$ and $\partial_{L} \varphi(x)$ is the limiting subdifferential of $\varphi$ at $x$ defined (for a lower semicontinuous function) by

$$
\partial_{L} \varphi(x):=\left\{\lim \xi_{i}: \xi_{i} \in \partial_{P} \varphi\left(x_{i}\right), x_{i} \longrightarrow x \text { and } \varphi\left(x_{i}\right) \longrightarrow \varphi(x)\right\} .
$$

For more informations about these definitions, see [10]. The following proposition gives a sufficient conditions for a continuous function to be mildly regular at a point $x$. For the proof, see [11], Proposition 6.3. 
Proposition 3.4. Let $\varphi$ be continuous in a neighborhood of a point $x$ and assume that one of the following condition holds:

(1) $\varphi$ is regular at $x^{7}$;

(2) $\varphi$ is differentiable at $x$;

(3) $\partial_{P} \varphi(x)$ is nonempty.

Then $\varphi$ is mildly regular at $x$.

Before giving some applications for the mildly regular property, let us give some geometric definitions. Let $A$ be a nonempty closed subset of $\mathbb{R}^{\mathrm{n}}$. For $\alpha \in \mathbb{R}^{\mathrm{n}}$, we denote by $\operatorname{proj}_{A}(\alpha)$ the set of closest points of $\alpha$ onto $A$ (that is, the set of points $\beta \in A$ such that $d_{A}(\alpha)=\|\alpha-\beta\|$ ). Now let $\beta \in A$. We define the proximal normal cone to $A$ at $\beta$ by

$$
N_{A}^{P}(\beta):=\left\{t(\alpha-\beta): t \geq 0 \text { and } \beta \in \operatorname{proj}_{A}(\alpha)\right\}
$$

We also define the generalized exterior normal to $A$ at $\beta$ by

$$
N_{A}(\beta):=\left\{\frac{\xi}{\|\xi\|}: \xi \in N_{A}^{P}(\beta) \backslash\{0\}\right\} .
$$

For more information about these definitions, see [10,21]. The following theorem gives a necessary condition for $-T(S, \cdot)$ to be the minimal solution of $\left(\mathrm{HJ}_{S}\right)$.

Theorem 3.5. Assume that the function $T(S, \cdot)$ is mildly regular on $\mathcal{R}_{+}^{S} \backslash S$. Assume further that $\operatorname{dom} N_{S}^{P}:=$ $\left\{s \in S: N_{S}^{P}(s) \neq\{0\}\right\}=\partial S^{8}$. Then the minimal solution $\varphi_{S}$ coincides with $-T(S, \cdot)$ and then every point of $\mathcal{R}_{+}^{S}$ lies on a semigeodesic $x$ from $S$ which satisfies $T(S, x(t))=t$ for all $t \geq 0$.

Proof. It is sufficient to prove that $-T(S, \cdot)$ is a solution of $\left(\mathrm{HJ}_{S}\right)$. By the definition, we have $-T(S, x)=0$ for all $x \in S$. Let us show that $-T(S, \cdot)$ satisfies the Hamilton-Jacobi equation of $\left(\mathrm{HJ}_{S}\right)$. Let $\alpha \in \mathcal{R}_{+}^{S}$, then there exist two cases.

Case 1. $\alpha \notin S$.

Let $\zeta \in \partial_{P}(-T(S, \cdot))(\alpha)$. Then $-\zeta \in \partial^{P} T(S, \cdot)(\alpha) \subset \partial_{L} T(S, \cdot)(\alpha)$, since $T(S, \cdot)$ is mildly regular at $\alpha$. But it is well-known that we have

$$
1+h_{-F}\left(\alpha, \partial_{P} T(S, \cdot)(\alpha)\right)=0^{9} .
$$

Hence since $h_{-F}$ is continuous and $\partial_{L}$ is constructed from $\partial_{P}$ by a limiting process we get that $1+h_{-F}(\alpha,-\zeta)=0$, and then $1+h(\alpha, \zeta)=0$.

Case 2. $\alpha \in S$.

We claim that $\partial_{P}(-T(S, \cdot))(\alpha)=\emptyset$. Indeed, if not then since $0 \in \partial_{P} T(S, \cdot)(\alpha)$ we get that $T(S, \cdot)$ is differentiable at $\alpha$ and $\partial_{P} T(S, \cdot)(\alpha)=\{0\}$. But by [22], Theorem 5.1, we have $\partial_{P} T(S, \cdot)(\alpha)=N_{S}^{P}(\alpha) \cap\left\{\zeta \in \mathbb{R}^{\mathrm{n}}\right.$ : $h(x, \zeta) \geq-1\}$, then since $N_{S}^{P}(\alpha) \neq\{0\}$ we find a contradiction.

Remark 3.6. We remark that in the preceding proof, we proved directly that $-T(S, \cdot)$ is a (minimal) solution of $\left(\mathrm{HJ}_{S}\right)$ and then we can eliminate the hypothesis $\mathcal{G}_{S} \neq \emptyset$ in Theorem 3.5.

Corollary 3.7. Let $F$ admit a representation of the form $F(x)=\{A x+u: u \in U\}$, where $A$ is an $n \times n$ matrix and $U$ is a compact and convex set. Assume further that we have the following hypotheses:

- $S$ is a convex set;

- for all $\alpha \in S$ and for all unit vector $\gamma \in N_{S}^{P}(\alpha)$, we have $h(\alpha, \gamma)<0^{10}$.

\footnotetext{
$7 \varphi$ is regular in the sense of Clarke, see [10].

8 A sufficient condition for $\operatorname{dom} N_{S}^{P}=\partial S(=S$ since int $S=\emptyset)$ is for example $S=\partial A$, where $A$ is a compact and convex set.

9 This is a well-known characterization of the minimal time function but here applied for the dynamic $-F$. We note that $h_{-F}$ is the lower Hamiltonian for the dynamic $-F$.

10 This condition reduces to $0 \in \operatorname{int} F(\alpha)$ if $S=\{\alpha\}$. For more informations about this hypothesis see [3].
} 
Then $\varphi_{S}$ is semiconcave and coincides with $-T(S, \cdot)$, and then every point of $\mathcal{R}_{+}^{S}$ lies on a semigeodesic $x$ from $S$ which satisfies $T(S, x(t))=t$ for all $t \geq 0$.

Proof. Follows from Theorem 3.5 and using [5], Theorem 4.1, which asserts that in this case the function $T(S, \cdot)$ is semiconvex on $\mathcal{R}_{+}^{S}$ and then mildly regular on $\mathcal{R}_{+}^{S}$ (for more informations about semiconcave and semiconvex functions, see $[6])$.

\section{Eikonal EQUations}

In this section we apply the results of the preceding sections to eikonal equations. We consider the following global eikonal equation:

$$
\left\|\partial_{P} \varphi(x)\right\|=1 \quad \forall x \in \mathbb{R}^{\mathrm{n}}, \quad \varphi(S)=0 . \quad\left(\mathrm{e}_{S}\right)
$$

We remark that $\left(\mathrm{e}_{S}\right)$ is exactly the Hamilton-Jacobi equation $\left(\mathrm{HJ}_{S}\right)$ corresponding to the choice $\bar{B}$ of $F$ (that is, $F(x)=\bar{B}$ for all $\left.x \in \mathbb{R}^{\mathrm{n}}\right)$. Clearly in this case we have:

- $F$ and $-F$ are $\beta$-STLC for all $\beta \in \mathbb{R}^{\mathrm{n}}$ (since $0 \in \operatorname{int} F(\beta)$ ).

- $\mathcal{R}=\mathbb{R}^{\mathrm{n}} \times \mathbb{R}^{\mathrm{n}}$ and $T(\alpha, \beta)=\|\alpha-\beta\|$ for all $(\alpha, \beta) \in \mathbb{R}^{\mathrm{n}} \times \mathbb{R}^{\mathrm{n}}$.

- $T(S, \cdot)=T(\cdot, S)=d_{S}(\cdot)$, where $d_{S}(\cdot)$ is the distance function associated to $S$.

- The minimal trajectory between two points $\alpha$ and $\beta$ is exactly the segment $[\alpha, \beta]$.

- If $\varphi$ is a solution of $\left(\mathrm{e}_{S}\right)$ then $\operatorname{dom} \varphi=\mathbb{R}^{\mathrm{n}}$.

Then we have the following corollary.

Corollary 4.1. The following statements are equivalent:

(i) The equation $\left(\mathrm{e}_{S}\right)$ admits a solution.

(ii) For all $\alpha \in S$ there exists a unit vector $\lambda \in \mathbb{R}^{\mathrm{n}}$ such that

$$
d_{S}(\alpha+t \lambda)=t \quad \forall t \geq 0 .
$$

(iii) $S \subset \partial(\operatorname{co} S)$.

Proof. The equivalence $(i) \Longleftrightarrow($ ii $)$ follows from Corollary 2.6 and the equivalence $(i i) \Longleftrightarrow$ (iii) follows from the following lemma (we omit the proof):

Lemma 4.2. Let $\alpha \in S$. Then the following assertions are equivalent.

- There exists a unit vector $\lambda \in \mathbb{R}^{\mathrm{n}}$ such that $d_{S}(\alpha+t \lambda)=t \quad \forall t \geq 0$.

- $\alpha \in \partial(\operatorname{co} S)$.

Example 4.3. We take $n=2$, and we consider the following data:

- $F(x, y):=\bar{B}$ for all $(x, y) \in \mathbb{R}^{2}$.

- $\alpha:=(0,1), \beta:=(1,0)$ and $\gamma:=(-1,0)$.

- $S:=[\alpha, \beta] \cup[\alpha, \gamma]$, where $[\alpha, \beta]$ (resp. $[\alpha, \gamma]$ ) is the segment joining $\alpha$ to $\beta$ (resp. $\alpha$ to $\gamma$ ).

Clearly we have $S \subset \partial(\operatorname{co} S)$. Then by Corollary 4.1, the Hamilton-Jacobi equation $\left(\mathrm{HJ}_{S}\right)$ admits a solutions. Let us calculate a solution using Proposition 2.8. Let $(a, b) \in[\alpha, \gamma]$. We denote by $z$ the exterior perpendicular to $[\alpha, \gamma]$ at $(a, b)$ (see Fig. 1$)$. Clearly $z$ is a semigeodesic from $(a, b)$ which satisfies $T(S, z(t))=t \forall t \geq 0$. We calculate $z$ and we find that

$$
z(t)=\left(-\frac{\sqrt{2}}{2} t+a, \frac{\sqrt{2}}{2} t+b\right) \quad t \geq 0
$$

Now let $\Gamma_{1}:=\{z(t): t \geq 0\}$, then by Proposition 2.8 we have that $\varphi_{\Gamma_{1}}$ is a solution of $\left(\mathrm{HJ}_{(a, b)}\right)$ which satisfies $\varphi_{\Gamma_{1}}(S) \geq 0$. We calculate this function (using its definition) and we obtain that

$$
\varphi_{\Gamma_{1}}(x, y)=\frac{\sqrt{2}}{2}(x-y+1) \quad \forall(x, y) \in \mathbb{R}^{2} .
$$




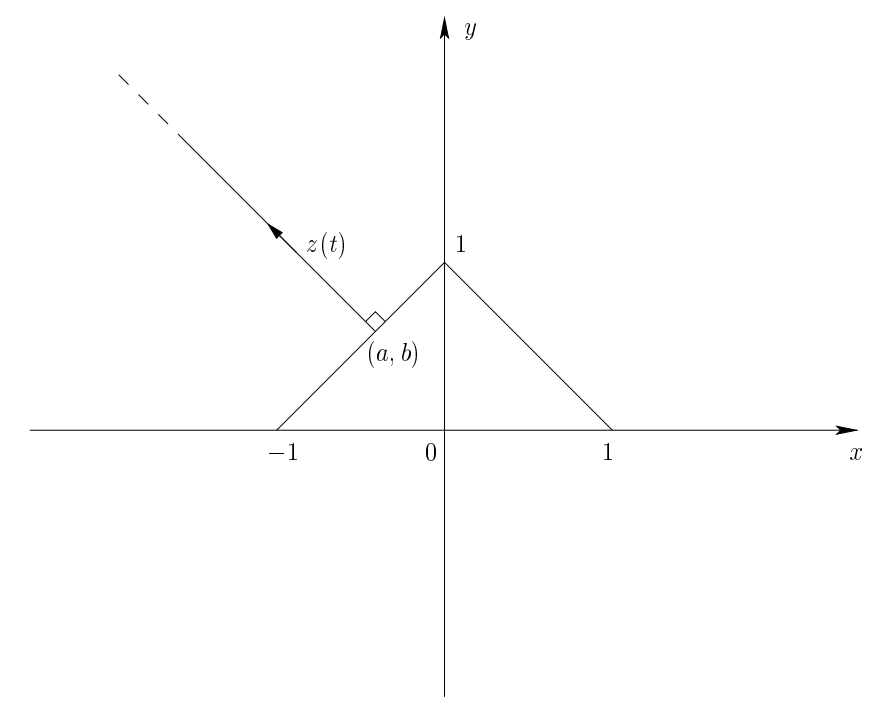

Figure 1. Example 4.3.

We remark that this function does not depend on $(a, b)$ and then it is a solution of $\left(\mathrm{HJ}_{(a, b)}\right)$ for all $(a, b) \in[\alpha, \gamma]$. Using the same idea, the following function

$$
\varphi_{\Gamma_{2}}(x, y):=\frac{\sqrt{2}}{2}(-x-y+1)
$$

is a solution of $\left(\mathrm{HJ}_{(a, b)}\right)$ for all $(a, b) \in[\alpha, \beta]$. Moreover as for $\varphi_{\Gamma_{1}}$, we have $\varphi_{\Gamma_{2}}(S) \geq 0$. Then by Proposition 2.8 , the function $\varphi:=\min \left\{\varphi_{\Gamma_{1}}, \varphi_{\Gamma_{2}}\right\}$ is a solution of $\left(\mathrm{HJ}_{S}\right)$.

We note that at the point $\alpha$, there exist several semigeodesics $z(t)$ satisfying $T(S, z(t))=t \forall t \geq 0$, and then we can construct (using the preceding algorithm) several solutions of $\left(\mathrm{HJ}_{S}\right)$.

Now we characterize, using the result of Section 3 , the minimal solution of $\left(\mathrm{e}_{S}\right)$.

Proposition 4.4. Assume that $S \subset \partial(\cos )$ and let $P_{S}:=\left\{\alpha \in \mathbb{R}^{\mathrm{n}} \backslash S: \operatorname{proj}_{(\cos S)}(\alpha) \cap S \neq \emptyset\right\}$. Then $\varphi_{S}(\alpha)=-d_{S}(\alpha)$ for all $\alpha \in P_{S}$. Moreover, for $\alpha \in P_{S}$ and $\beta \in \operatorname{proj}_{(\cos S)}(\alpha) \cap S\left(\beta\right.$ is unique) if $N_{(\cos )}(\beta)$ is a singleton then for all $\varphi$ a solution of $\left(e_{S}\right)$, we have $\varphi(\alpha)=-d_{S}(\alpha)$.

Proof. Let $\alpha \in P_{S}$. Then there exists (a unique) $\beta \in S$ such that $d_{(\operatorname{co} S)}(\alpha)=d_{S}(\alpha)=\|\alpha-\beta\|$. We set $\lambda:=\frac{\alpha-\beta}{\|\alpha-\beta\|}$ and we consider the trajectory $x(t):=\beta+\lambda t$ for all $t \geq 0$. Clearly the trajectory $x$ is a semigeodesic from $S$ passing through $\alpha$. Moreover, we can easily prove that

$$
d_{(\operatorname{co} S)}(x(t))=d_{S}(x(t))=t \quad \forall t \geq 0 .
$$

But $T(S, \cdot)=T(\cdot, S)=d_{S}(\cdot)$, then by Theorem 3.2 we get that

$$
\varphi_{S}(\alpha)=-T(S, \alpha)=-d_{S}(\alpha)
$$

Now we take $\alpha \in P_{S}$ and we assume that for (the unique) $\beta \in \operatorname{proj}_{(\operatorname{co} S)}(\alpha) \cap S$ we have that $N_{(\operatorname{co} S)}(\beta)$ is a singleton. Let $\varphi$ be a solution of $\left(\mathrm{e}_{S}\right)$. We consider $\lambda$ and $x(\cdot)$ as above. Since $\beta \in S$ and by Proposition 2.2 there exists a semigeodesic $y(t)=\beta+\lambda^{\prime} t\left(\left\|\lambda^{\prime}\right\|=1\right)$ form $\beta$ such that

$$
\varphi(y(t))+t=0 \text { and } d_{S}(y(t))=T(S, y(t))=t \quad \forall t \geq 0 .
$$


Using the fact that $d_{S}(y(t))=t$ for all $t \geq 0$, we can prove that $\lambda^{\prime} \in N_{\operatorname{coS}}(\alpha)$. Then $\lambda^{\prime}=\lambda$ and this gives that $y$ coincides with $x$. Hence

$$
\varphi(\beta+\lambda t)+t=0 \quad \forall t \geq 0 .
$$

Taking $t=\|\alpha-\beta\|$, we get that

$$
\varphi(\alpha)=-\|\alpha-\beta\|=-d_{S}(\alpha)
$$

Theorem 4.5. Assume that $S=\partial(\operatorname{co} S)$. Then we have the following:

(i) If int $(\operatorname{co} S)=\emptyset$ then $\varphi_{S}(\cdot)=-d_{S}(\cdot)$.

(ii) If int $(\cos S) \neq \emptyset$ then

$$
\varphi_{S}(\alpha)= \begin{cases}-d_{S}(\alpha) & \text { if } \alpha \in \mathbb{R}^{\mathrm{n}} \backslash \operatorname{co} S \\ 0 & \text { if } \alpha \in S \\ d_{S}(\alpha) & \text { if } \alpha \in \operatorname{int}(\operatorname{co} S)\end{cases}
$$

Proof.

(i) Follows from Corollary 3.7.

(ii) By Proposition 4.4, we have that $\varphi_{S}(\cdot)=-d_{S}(\cdot)$ on $\mathbb{R}^{\mathrm{n}} \backslash \operatorname{co} S$. Let us prove that $\varphi_{S}(\cdot)=d_{S}(\cdot)$ on int $(\operatorname{co} S)$. Let $\alpha \in \operatorname{int}(\operatorname{co} S)$, then by Proposition 2.2 there exists a semigeodesic $x(t)=\alpha+t \lambda(\|\lambda\|=1)$ from $\alpha$ such that

$$
\varphi_{S}(x(t))+t=\varphi_{S}(\alpha) \quad \forall t \geq 0 .
$$

Clearly there exists $t_{0}>0$ such that $x\left(t_{0}\right)=\beta \in S$. Then

$$
\varphi_{S}(\beta)+t_{0}=\varphi_{S}(\alpha) .
$$

But we can easily prove that $t_{0}=\|\alpha-\beta\|$, hence

$$
\|\alpha-\beta\|=\varphi_{S}(\alpha)
$$

On the other hand and by Proposition 2.2, we have that $\varphi_{S}(\alpha) \leq d_{S}(\alpha) \leq\|\alpha-\beta\|$. This gives that $\varphi_{S}(\alpha)=d_{S}(\alpha)$ which completes the proof.

Corollary 4.6. Assume that $S=\partial(\operatorname{co} S)$ and that $N_{(\operatorname{co} S)}(\beta)$ is a singleton for all $\beta \in S$. Then the function $\varphi_{S}$ defined in $(*)$ is the unique solution of $\left(e_{S}\right)$.

Proof. This follows from Theorem 4.5 and from the fact that if $N_{(\operatorname{co} S)}(\beta)$ is a singleton for all $\beta \in S$, then $\operatorname{int}(\operatorname{co} S) \neq \emptyset$.

Remark 4.7. In Theorem 4.5, we can prove that $\varphi_{S}(\cdot)=d_{S}(\cdot)$ on int $(\operatorname{co} S)$ using the following known result (see $[3])$ :

Theorem 4.8. Let $\Omega$ be a nonempty bounded open set of $\mathbb{R}^{\mathrm{n}}$. Then the function $d_{\partial \Omega}(\cdot)$ is the unique solution of the following eikonal equation

$$
\left\|\partial_{P} \varphi(\alpha)\right\|=1 \quad \forall \alpha \in \Omega, \quad \varphi=0 \text { on } \partial \Omega
$$




\section{Geodesics}

A trajectory $x:]-\infty,+\infty\left[\longrightarrow \mathbb{R}^{\mathrm{n}}\right.$ of $F$ is a geodesic if and only if

$$
T(x(s), x(t))=t-s \quad \forall s \leq t \in]-\infty,+\infty[
$$

We are interested in this section by the geodesics passing through $S$ (that is $x(0) \in S$ ) and which satisfy the following

$$
T(x(-t), S)=T(S, x(t))=t \quad \forall t \geq 0 .
$$

We remark that if $x$ is a geodesic passing through $S$ which satisfies $(+)$, then $x$ intersects $S$ only on one point which is $x(0)$. Clearly under our hypotheses, we cannot guarantee the existence of such geodesic (see the case $S_{1}=\left\{-\frac{1}{2}, \frac{1}{2}\right\}$ in Ex. 2.7). To study the existence of this type of geodesics, we define the following dual equation of $\left(\mathrm{HJ}_{S}\right)$ :

$$
1+h\left(x, \partial^{P} \varphi(x)\right)=0 \quad \forall x \in \mathcal{R}_{-}^{S}, \varphi(S)=0 . \quad\left(\mathrm{HJ}_{S}^{*}\right)
$$

A solution of $\left(\mathrm{HJ}_{S}^{*}\right)$ refers to an upper semicontinuous function. We remark that a function $\psi$ is a solution of $\left(\mathrm{HJ}_{S}^{*}\right)$ if and only if the function $\varphi=-\psi$ is a solution of the version of $\left(\mathrm{HJ}_{S}\right)$ obtained by replacing $F$ by $-F$. Since the trajectories of $-F$ correspond to trajectories of $F$ in reversed time, we can apply our previous results to $\left(\mathrm{HJ}_{S}^{*}\right)$ but in this case we replace the trajectories of $F$ on $[0,+\infty[$ by trajectories on $]-\infty, 0]($ past rather than future time).

Theorem 5.1. Let $\alpha \in S$ and assume that the following hypotheses hold:

- $F$ and $-F$ are $\alpha$-LC.

- $S \subset \mathcal{R}_{-}^{\alpha} \subset \mathcal{R}_{+}^{\alpha}$.

- There exist solution $\varphi$ and $\psi$ of $\left(\mathrm{HJ}_{\alpha}\right)$ and $\left(\mathrm{HJ}_{\alpha}^{*}\right)$ respectively such that

- $\varphi(S) \geq 0$.

- $\psi(S) \leq 0$.

- $\varphi \geq \psi$ on $\mathcal{R}_{-}^{\alpha}$

Then there exists a geodesic passing through $S$ at $\alpha(x(0)=\alpha)$ and satisfying $(+)$.

Proof. By Proposition 2.8 there exists a trajectory $x$ with $x(0)=\alpha$ such that

$$
\varphi(x(t))+t=0 \text { and } T(S, x(t))=t \quad \forall t \geq 0,
$$

and a trajectory $y$ with $y(0)=\alpha$ such that

$$
\psi(y(t))+t=0 \text { and } T(x(t), S)=-t \quad \forall t \leq 0 .
$$

Then since $\varphi \geq \psi$ on $\mathcal{R}_{-}^{\alpha}$, we get that

$$
\varphi(y(t))+t \geq 0 \quad \forall t \leq 0
$$

But the opposite inequality holds by strong increase. Then the trajectory $z$ defined on $]-\infty,+\infty[$ by concatenating $y$ and $x$ satisfies

$$
\varphi(z(t))+t=0 \quad \forall t \in \mathbb{R} \text { and } T(z(-t), S)=T(S, z(t))=t \quad \forall t \geq 0 .
$$

This gives, using Proposition 2.1, that $z$ is a geodesic passing through $S$ at $\alpha$ and satisfying $(+)$.

Theorem 5.2. Assume that the following hypotheses hold:

- $F$ is $S$-LC.

- $\mathcal{R}_{-}^{S} \subset \mathcal{R}_{+}^{S}$.

- There exists solutions $\varphi$ and $\psi$ of $\left(\mathrm{HJ}_{S}\right)$ and $\left(\mathrm{HJ}_{S}^{*}\right)$ respectively such that $\varphi \geq \psi$ on $\mathcal{R}_{-}^{S}$.

Then for all $\alpha \in S$ there exists a geodesic passing through $S$ at $\alpha(x(0)=\alpha)$ and satisfying $(+)$. 
Proof. Let $\alpha \in S$. Then by Proposition 2.2 there exists a trajectory $x$ with $x(0)=\alpha$ such that

$$
\varphi(x(t))+t=0 \text { and } T(S, x(t))=t \quad \forall t \geq 0,
$$

and a trajectory $y$ with $y(0)=\alpha$ such that

$$
\psi(y(t))+t=0 \text { and } T(x(t), S)=-t \quad \forall t \leq 0 .
$$

Then we continue as in the proof of Theorem 5.1.

Corollary 5.3. Assume that the following hypotheses hold:

- $F$ is is $S$-LC.

- $\mathcal{R}_{-}^{S} \subset \mathcal{R}_{+}^{S}$.

- There exists a continuous solution $\varphi$ of $\left(\mathrm{HJ}_{S}\right)$ which is mildly regular on $\mathcal{R}_{+}^{S}$.

Then for all $\alpha \in S$ there exists a geodesic passing through $S$ at $\alpha$ and satisfying $(+)$.

Proof. The function $\varphi$ satisfies

$$
1+h\left(x, \partial_{L} \varphi(x)\right)=0 \quad \forall x \in \mathcal{R}_{+}^{S}, \varphi(S)=0,
$$

since $h$ is continuous, and since $\partial_{L}$ is constructed from $\partial_{P}$ by a limiting process. Then since $\varphi$ is mildly regular on $\mathcal{R}_{+}^{S}$, we get that $\varphi$ is a solution of $\left(\mathrm{HJ}_{S}^{*}\right)$. The result follows from Theorem 2.8 .

We denote by $E_{S}(\cdot)$ the pointwise upper envelope of all solutions of $\left(\mathrm{HJ}_{S}\right)$. This is a lower semicontinuous function bounded above by $T(\cdot, S)$. In the following theorem, we use this function to give a necessary and sufficient condition for the existence of geodesic passing through $S$ and satisfying $(+)$.

Theorem 5.4. Assume that the following hypotheses hold:

- $\mathcal{R}_{-}^{S} \subset \mathcal{R}_{+}^{S}$.

- $F$ and $-F$ are $\beta$-STLC for all $\beta \in \mathcal{R}_{-}^{S 11}$.

Then we have the following:

(a) $\limsup \left[E_{S}(\beta)-T(\beta, S)\right] \geq 0 \Longrightarrow$ there exist a geodesic passing through $S$ and satisfying $(+)$.

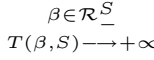

(b) For $\alpha \in S$ we have:

(i) There exists a geodesic passing through $S$ at $\alpha$ and satisfying $(+) \Longrightarrow \limsup _{\substack{\beta \in \mathcal{R}_{-}^{\alpha} \\ T(\beta, S) \rightarrow+\infty}}\left[E_{\alpha}(\beta)-T(\beta, S)\right] \geq 0$.

(ii) $\limsup _{\beta \in \mathcal{R}^{\alpha}}\left[E_{S}(\beta)-T(\beta, \alpha)\right] \geq 0 \Longrightarrow$ there exists a geodesic passing through $S$ at $\alpha$ and satisfying $(+)$. $\underset{T(\beta, \alpha) \rightarrow+\mathcal{R}^{\alpha}}{\rightarrow}+\infty$

Proof. (a) Assume that

$$
\limsup _{\substack{\beta \in \mathcal{R}_{-} \\ T(\beta, S) \rightarrow+\infty}}\left[E_{S}(\beta)-T(\beta, \alpha)\right] \leq 0 .
$$

Then since $E_{S}(\cdot) \leq T(\cdot, S)$ we get that

$$
\limsup _{\beta \in \mathcal{R} \underline{-}}\left[E_{S}(\beta)-T(\beta, \alpha)\right]=0 .
$$

${ }^{11}$ Since $\mathcal{R}_{-}^{S} \subset \mathcal{R}_{+}^{S}$ and using [17], Proposition 4.2, we can show that this condition is equivalent to the continuity of $T(\cdot, \cdot)$ on $\mathcal{R}_{-}^{S} \times \mathcal{R}_{+}^{S}$. 
Hence, there exists $\varepsilon_{i} \longrightarrow 0$ and $\beta_{i}$ be a sequence in $\mathcal{R}_{-}^{S}$ such that

- $T\left(\beta_{i}, S\right) \longrightarrow+\infty$ and $E_{S}\left(\beta_{i}\right)-T\left(\beta_{i}, S\right) \longrightarrow 0$.

- For each $i$ there exists a solution $\varphi_{i}$ of $\left(\mathrm{HJ}_{S}\right)$ which satisfies

$$
\varphi_{i}\left(\beta_{i}\right) \geq T\left(\beta_{i}, S\right)-\varepsilon_{i}
$$

Set $\tau_{i}=T\left(\beta_{i}, S\right)$, and let $x_{i}$ be an optimal trajectory on the interval $\left[-\tau_{i}, 0\right]$ joining $\beta_{i}$ to $S$. Let $\alpha_{i}:=x_{i}(0)$, then since $S$ is compact we can assume that $\alpha_{i} \longrightarrow \alpha \in S$. By Proposition 2.2 we can extend $x_{i}$ to [0, $+\infty$ [ by a trajectory satisfying

$$
\varphi_{i}\left(x_{i}(t)\right)+t=0 \quad \text { and } T\left(S, x_{i}(t)\right)=t \quad \forall t \geq 0 .
$$

Since $x_{i}(t) \in \mathcal{R}_{-}^{S} \subset \mathcal{R}_{+}^{S} \forall t \in\left[-\tau_{i}, 0\right]$ and by the strong increasing property, we have that for any $t \in\left[-\tau_{i}, 0\right]$,

$$
\begin{aligned}
0=\varphi\left(x_{i}(0)\right)+0 & \geq \varphi_{i}\left(x_{i}(t)\right)+t \\
& \geq \varphi_{i}\left(x_{i}\left(-\tau_{i}\right)\right)-\tau_{i} \\
& =\varphi_{i}\left(\alpha_{i}\right)-T\left(\alpha_{i}, 0\right) \\
& \geq-\varepsilon_{i} .
\end{aligned}
$$

We deduce that

$$
-\varepsilon_{i} \leq \varphi_{i}\left(x_{i}(t)\right)+t \leq T\left(x_{i}(t), S\right)+t \leq 0 \quad \forall t \in\left[-\tau_{i}, 0\right] .
$$

By (4), (5) and Proposition 2.1, we get that for any two points $s \leq t \in\left[-\tau_{i},+\infty\right.$ [ we have

$$
t-s \geq T\left(x_{i}(s), x_{i}(t)\right) \geq t-s-\varepsilon_{i}
$$

By the compactness property of trajectories, we can assume that the sequence $x_{i}$ converges uniformly on bounded intervals to a trajectory $x$. Clearly we have $x(0)=\alpha \in S$. We claim that $x$ is the researched geodesic. Indeed, let $s \in]-\infty, 0]$ and let $t \in\left[0,+\infty\left[\right.\right.$. We have $x(s) \in \mathcal{R}_{-}^{S}$ and $x(t) \in \mathcal{R}_{+}^{S}$. Then $T(\cdot, \cdot)$ is continuous at $(x(s), x(t))$ and by $(6)$ we get that

$$
T(x(s), x(t))=t-s,
$$

which gives that $x$ is a geodesic. Using the continuity of $T(S, \cdot)$ and $T(\cdot, S)$ and by $(4)$ and $(5)$, we get that $x$ satisfies $(+)$.

(b) $(i)$ Let $\alpha \in S$ and assume that there exists a geodesic $x$ passing through $S$ at $\alpha$ and satisfying $(+)$. We define $\Gamma:=\{x(t): t \geq 0\}$, and we consider the solution $\varphi_{\Gamma}$ of $\left(\mathrm{HJ}_{\alpha}\right)$. Set $\beta_{i}:=x(-i)$. Then for any $\beta \in \mathcal{R}_{+}^{\alpha}$ and for $t>0$ we have

$$
\begin{aligned}
T(\beta, x(t))-T(\alpha, x(t)) & =T\left(\beta_{i}, x(t)\right)-T(S, x(t))+T(\beta, x(t))-T\left(\beta_{i}, x(t)\right) \\
& \geq T\left(\beta_{i}, S\right)-T\left(\beta_{i}, \beta\right) .
\end{aligned}
$$

Using the definition of $\varphi_{\Gamma}$ and since $T(\cdot, \cdot)$ is continuous at $\left(\beta_{i}, \beta_{i}\right) \in \mathcal{R}_{-}^{S} \times \mathcal{R}_{+}^{S}$ we get that

$$
\varphi_{\Gamma}\left(\beta_{i}\right) \geq T\left(\beta_{i}, S\right)=T\left(\beta_{i}, \alpha\right)
$$

Then $E_{\alpha}\left(\beta_{i}\right)=T\left(\beta_{i}, S\right)$. Since $T\left(\beta_{i}, S\right) \longrightarrow+\infty$ and $\beta_{i} \in \mathcal{R}_{-}^{S}$, the result follows.

(ii) Follows using the same idea as in $(a)$. 
Remark 5.5. If we assume that $S=\left\{\alpha_{0}\right\}$ in the preceding theorem, then we obtain that

$$
\limsup _{\substack{\beta \in \mathcal{R}_{-}^{\alpha_{0}} \\ T\left(\beta, \alpha_{0}\right) \longrightarrow+\infty}}\left[E_{\alpha_{0}}(\beta)-T\left(\beta, \alpha_{0}\right)\right]=0 \Longleftrightarrow \text { there exists a geodesic passing through } \alpha_{0} \text {. }
$$

Then Theorem 5.4 generalizes [11], Theorem 8.6.

Acknowledgements. We would like to thank Francis Clarke for his comments and suggestions. We also thank Ludovic Rifford for fruitful discussions. Finally, I'm grateful to the referees who read the paper with great care and made interesting remarks.

\section{REFERENCES}

[1] O. Alvarez, S. Koike and I. Nakayama, Uniqueness of lower semicontinuous viscosity solutions for the minimum time problem. SIAM J. Control Optim. 38 (2000) 470-481.

[2] J.P. Aubin and A. Cellina, Differential inclusions. Springer-Verlag, New York (1984).

[3] M. Bardi and I. Capuzzo-Dolcetta, Optimal control and viscosity solutions of Hamilton-Jacobi-Bellman equations. With appendices by Maurizio Falcone and Pierpaolo Soravia. Birkhäuser Boston, Inc., Boston, MA (1997).

[4] E.N. Barron and R. Jensen, Semicontinuous viscosity solutions for Hamilton-Jacobi equations with convex Hamiltonians. Commun. Partial Differ. Equations 15 (1990) 1713-1742.

[5] P. Cannarsa and C. Sinestrari, Convexity properties of the minimum time function. Calc. Var. 3 (1995) 273-298.

[6] P. Cannarsa and C. Sinestrari, Semiconcave functions, Hamilton-Jacobi equations and optimal control problems. Birkhäuser Boston (2004).

[7] P. Cardaliaguet, M. Quincampoix and P. Saint-Pierre, Optimal times for constrained nonlinear control problems without local controllability. Appl. Math. Optim. 36 (1997) 21-42.

[8] F.H. Clarke and Yu. Ledyaev, Mean value inequalities in Hilbert space. Trans. Amer. Math. Soc. 344 (1994) 307-324.

[9] F.H. Clarke, Yu. Ledyaev, R. Stern and P. Wolenski, Qualitative properties of trajectories of control systems: A survey. J. Dynam. Control Syst. 1 (1995) 1-48.

[10] F.H. Clarke, Yu. Ledyaev, R. Stern and P. Wolenski, Nonsmooth Analysis and Control Theory. Graduate Texts Math. 178 (1998). Springer-Verlag, New York.

[11] F.H. Clarke and C. Nour, The Hamilton-Jacobi equation of minimal time control. J. Convex Anal. 11 (2004) $413-436$.

[12] M.G. Crandall, H. Ishi and P.L. Lions, User's guide to the viscosity solutions of second order partial differential equations. Bull. Amer. Math. Soc. 27 (1992) 1-67.

[13] M.G. Crandall and P.L. Lions, Viscosity solutions of Hamilton-Jacobi equations. Trans. Amer. Math. Soc. 277 (1983) 1-42.

[14] W.H. Fleming and H.M. Soner, Controlled Markov Processes and Viscosity Solutions. Springer-Verlag, New York (1993).

[15] H. Frankowska, Lower semicontinuous solutions of Hamilton-Jacobi-Bellman equations. SIAM J. Control Optim. 31 (1993) $257-272$.

[16] C. Nour, The Hamilton-Jacobi equation in optimal control: duality and geodesics. Ph.D. Thesis, Université Claude Bernard Lyon I (2003).

[17] C. Nour, The bilateral minimal time function. J. Convex Anal., to appear.

[18] P. Soravia, Discontinuous viscosity solutions to Dirichlet problems for Hamilton-Jacobi equations with convex Hamiltonians. Comm. Partial Differ. Equ. 18 (1993) 1493-1514.

[19] H.J. Sussmann, A general theorem on local controllability. SIAM J. Control Optim. 25 (1987) 158-133.

[20] V.M. Veliov, Lipschitz continuity of the value function in optimal control. J. Optim. Theory Appl. 94 (1997) 335-363.

[21] R.B. Vinter, Optimal control. Birkhäuser Boston, Inc., Boston, MA (2000).

[22] P. Wolenski and Y. Zhuang, Proximal analysis and the minimal time function. SIAM J. Control Optim. 36 (1998) $1048-1072$. 